\title{
Non-invasive detection of left atrial mechanical failure in patients with left ventricular disease
}

Ke Wang, Derek G Gibson

\begin{abstract}
Objective-To define patients with left atrial mechanical failure and identify its echocardiographic, physiological, and clinical associations.

Design-Prospective study with cross sectionally guided $M$ mode and Doppler echocardiograms, and with apexcardiograms, electrocardiograms, and phonocardiograms.
\end{abstract}

Setting-Tertiary cardiac referral centre. Patients-10 patients with left atrial mechanical failure and 20 healthy controls of similar age.

Results-10 patients with left atrial mechanical paralysis were identified among 4036 adults over a 1 year interval. Nine were in sinus rhythm and one had a DDD pacemaker. Left atrial mechanical activity was absent on $M$ mode echocardiograms of the left sided atrioventricular ring and the aortic root. $A$ Doppler $A$ wave on transmitral flow and a clearly defined $A$ wave on the left ventricular apexcardiogram were also absent, though evidence of right atrial mechanical movement was present in nine patients. Mean (SD) age was 63 (19) years and six were men. Nine had left ventricular disease and one had undergone extensive resection of the left atrium. Abnormal measurements of left ventricular end diastolic dimension (62 (13) $\mathrm{mm})$, fractional shortening (15 (6)\%), isovolumic relaxation time (19 (12) $\mathrm{ms}$ ), left atrial size (45 (10) $\mathrm{mm}$ ), and transmitral Doppler $\mathbf{E}$ wave deceleration time (110 (35) $\mathrm{ms})$ were recorded.

Conclusion-Left atrial mechanical failure may be present in patients with left ventricular disease despite normal sinus rhythm. Normal atrial activation on 12 lead electrocardiogram suggests it is primarily mechanical in origin. The possibility of left atrial mechanical failure must be considered when Doppler patterns of transmitral flow are used to assess left ventricular diastolic function.

Cardiac Department, Royal Brompton Hospital, London K Wang

D G Gibson

Correspondence to: Dr D G Gibson, Cardiac Department, Royal Brompton Hospital, Sydney Brompton Hospital, Sydney

Accepted for publication 26 April 1995
(Br Heart F 1995;74:536-540)

Keywords: left ventricular disease; atrial mechanical failure; echocardiography

An increased component of stroke volume entering the left ventricle during atrial systole is widely believed to be an important adaptation to left ventricular disease. Clinical deterioration occurring with the onset of atrial fibrillation is often attributed to its loss. In the present study we describe a group of patients with sinus rhythm in whom we were unable to detect any evidence of mechanical activity of the left atrium. As this abnormality has received little attention in the literature, and as loss of atrial function has obvious practical consequences, we discuss its diagnosis and clinical associations in a small series of patients.

\section{Patients and methods}

\section{PATIENTS}

Patients were identified among those studied in a single adult echocardiographic clinic over a period of 1 year. The series comprised 4036 patients in sinus rhythm confirmed by a standard 12 lead electrocardiogram (ECG) on the day of study. Evidence of absent mechanical activity of the left atrium was demonstrated in tests of atrial function, which included $M$ mode echocardiograms of left sided atrioventricular (AV) ring movement and aortic root, transmitral Doppler, and left ventricular apexcardiogram. Exclusory criteria included a heart rate above 110 beats/min, junctional nodal rhythm or any other significant arrhythmia, and a history of direct current shock for any indication within the previous 3 months. We ensured that any mechanical effect of atrial systole was not obscured by its being superimposed on early diastolic motion due to tachycardia, prolonged PR interval, or prolongation of early diastole itself.

\section{METHODS}

$M$ mode and cross sectional echocardiograms were taken with a Hewlett Packard model 77020 A Sonos 1000 echocardiograph and a $2.5 \mathrm{MHz}$ phased array transducer as the patients lay in the partial left lateral position. $M$ mode echocardiograms, Doppler transmitral and transtricuspid flow traces, and apexcardiograms were recorded separately on a Honeywell (Ecoline 22) strip chart recorder at a paper speed of $100 \mathrm{~mm} / \mathrm{s}$ with simultaneous ECG (lead II) and phonocardiogram.

Standard cross sectionally guided $M$ mode echocardiograms of the left ventricular minor axis were recorded at the level of the mitral leaflet. The timing of aortic valve closure was taken as the onset of the first high frequency component of the aortic valve closure sound (A2) and the isovolumic relaxation time as the time interval between this and the onset of 
mitral valve cusp separation. End systolic dimension was taken as the transverse diameter between the left ventricular septum and posterior wall at $\mathrm{A} 2$, and end diastolic dimension as the transverse diameter at the time of the onset of the $Q$ wave of the ECG. Aortic root echocardiograms were recorded at aortic cusp level to assess changes in left atrial transverse dimension. The presence of left atrial contraction was taken as discrete backward motion of the aortic posterior wall after the $P$ wave of the ECG. ${ }^{1}$ Motion of the AV rings during the cardiac cycle was recorded by $M$ mode echocardiography from the cross sectionally guided apical four chamber view, with the cursor passing longitudinally through the left, septal, and right sides of the AV rings. ${ }^{1}$ The

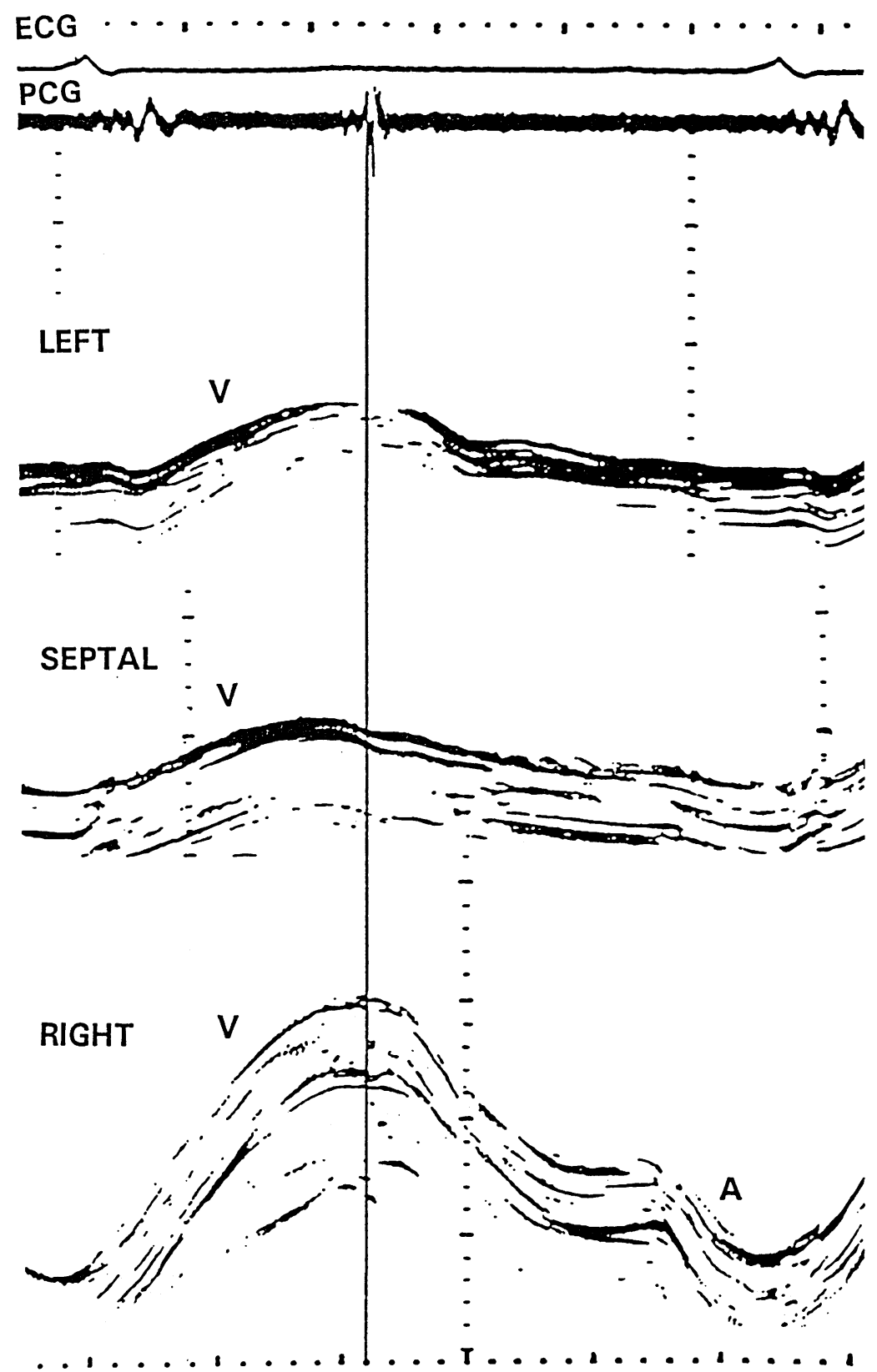

Figure $1 M$ mode echocardiograms of the atrioventricular $(A V)$ ring at the left, septal, and right sites in a patient with left atrial mechanical failure. Movement of the $A V$ ring represents mechanical activity of the atria $(A)$ and the ventricles $(V)$. Note that although the $A V$ ring remained stationary for a clearly defined period after the end of ventricular rapid early diastolic motion and before the start of next ventricular systolic motion, left atrial contraction was absent, while right atrial contraction is present. The vertical line corresponds to the second heart sound $\left(A_{2}\right)$. ECG, electrocardiogram; $P C G$, phonocardiogram. rings remained stationary in all patients for a clearly defined period after the end of ventricular rapid early diastolic motion and before the start of next ventricular systolic motion (fig 1). An A wave, if present, occurred after the $P$ wave on the ECG and was associated with further motion of the ring in the atrial duration.

Doppler transmitral and transtricuspid forward flow velocities were recorded at cusp tip level with Hewlett Packard or Doptek equipment in pulse mode $(2.0$ or $3.5 \mathrm{MHz}$ transducer). A wall filter corresponding to a velocity of $5 \mathrm{~cm} \cdot \mathrm{s}^{-1}$ was used. There was a clearly defined time interval between the end of the $\mathrm{E}$ wave and the onset of the succeeding systole in all patients (fig 2). An A wave was diagnosed as a discrete increase in blood flow velocity in this period, which occurred after the $P$ wave of the ECG.

Left ventricular apexcardiograms were recorded from the point of maximal impulse on the left side of the heart with the patient lying in the left lateral position. A Cambridge Instruments transducer was used with a time constant of $4 \mathrm{~s}$. An A wave, if present, was diagnosed as a discrete increase in displacement occurring after the $P$ wave of the ECG (fig 3).

\section{MEASUREMENTS}

Left ventricular end diastolic and end systolic dimensions, fractional shortening, isovolumic relaxation time (as A2 to mitral cusp separation), and left atrial size were measured from the original traces. On the pulsed Doppler traces of the transmitral and transtricuspid flow we measured peak $E$ and, if present, $A$ wave velocities and $A / E$ ratio. The overall amplitude of excursion during ventricular early diastolic lengthening, atrial contraction, and ventricular systolic shortening was measured from the AV ring movement traces. The presence or absence of movement corresponding to left atrial activity on the aortic root echocardiogram was sought after the $P$ wave of the ECG.

Twenty healthy controls of similar age, in whom there was no clinical, ECG, radiographic, or echocardiographic evidence of heart disease were also recruited to the study.

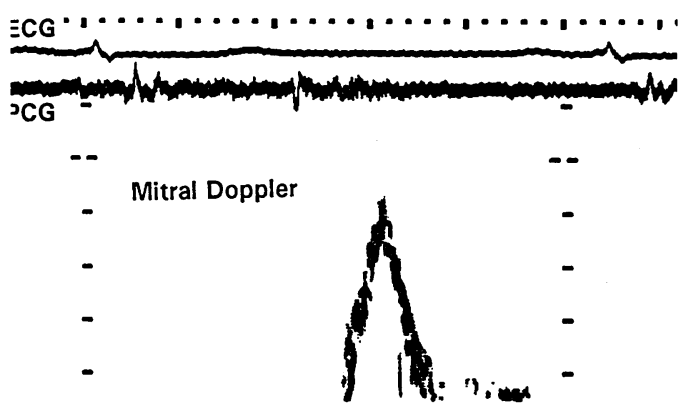

Figure 2 Mitral Doppler trace from a patient with left atrial mechanical failure. Note that an $A$ wave is absent, although there was a clearly defined time interval between the end of the $E$ wave and the onset of the next beat. Vertical scale: one division represents $20 \mathrm{~cm} / \mathrm{s}$. Abbreviations as in fig 1 . 
A

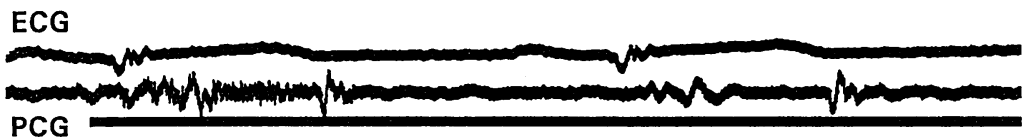

PCG

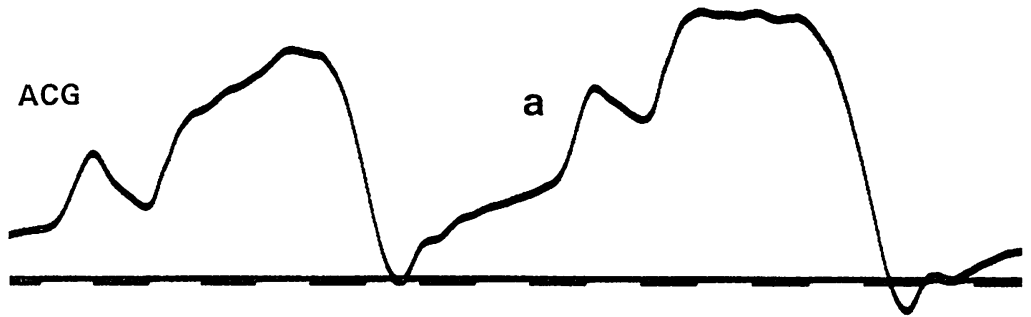

B

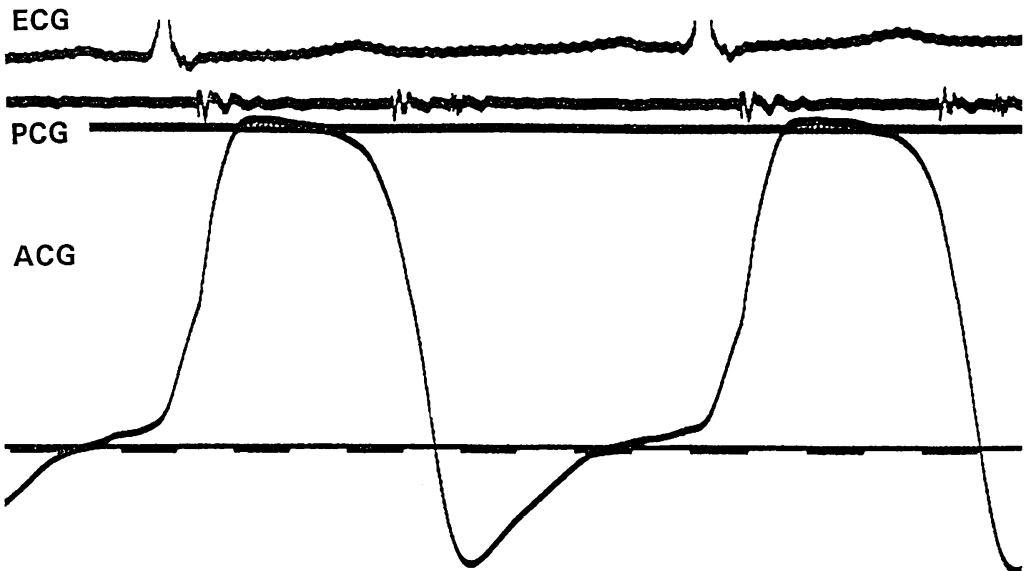

Figure 3 Apexcardiograms (ACG) from patients with $(A)$ restrictive left ventricular disease (not included in the present series) and (B) left atrial mechanical failure. Note the large $A$ wave (a) in the former and the lack of an $A$ wave in the latter patient. Abbreviations as in fig 1 .
DATA ANALYSIS

Average values were taken from three successive heart beats. Group measurements are expressed as mean (SD). Unpaired student's $t$ test was used as appropriate. A p value $<0.05$ was considered significant.
Table 1 Echocardiographic data

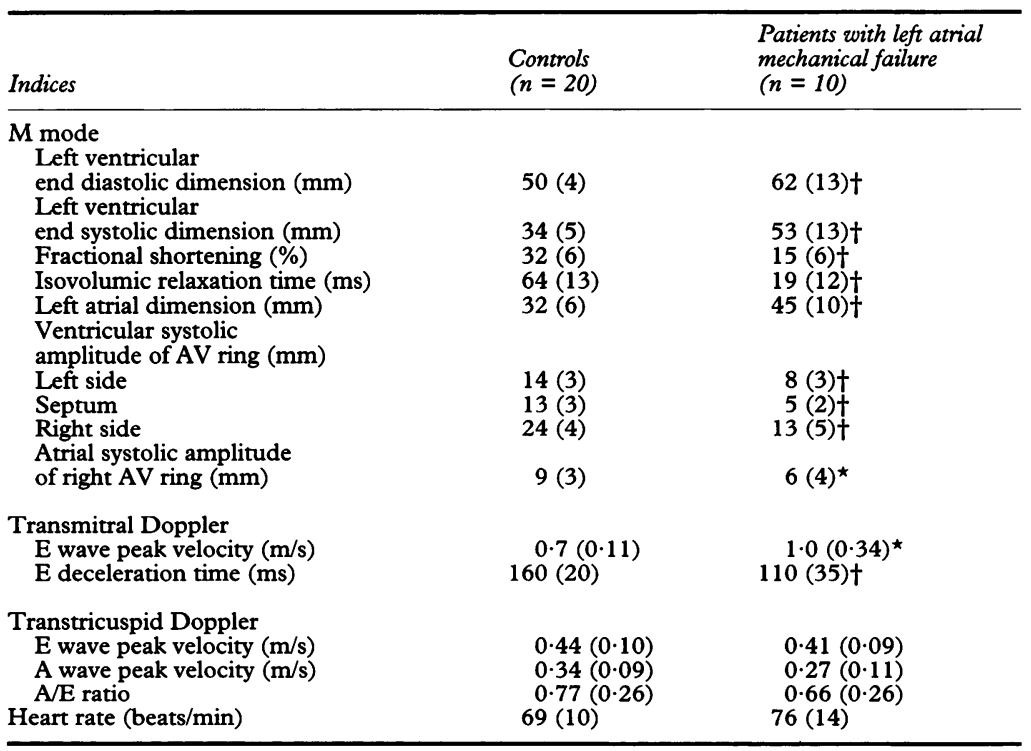

Values are mean $(\mathrm{SD})$; ${ }^{\star} \mathrm{P}<0.01 ;+\mathrm{P}<0.001$ versus controls; $\mathrm{AV}$, atrioventricular.
Results

PATIENTS

Ten of a total of 4036 patients studied fulfilled the criteria for absent mechanical activity of the left atrium. The (SD) mean (range) age was 63 (19) (31-80) years; six were men. Five patients had dilated cardiomyopathy, defined as an end diastolic left ventricular dimension of more than $6.5 \mathrm{~cm}$ with fractional shortening less than $15 \%$. Three patients had left ventricular hypertrophy, one with aortic stenosis and two with hypertrophic cardiomyopathy, one of whom had a DDD pacemaker. One patient had undergone mitral valve replacement, and in a further patient extensive resection of the left atrium was performed due to invasion of the heart by bronchogenic carcinoma. In no patient did the echocardiogram suggest amyloid disease.

The mean (SD) age of the control group was 57 (18) years; 11 were men. Age and gender were not significantly different from those of the patients with left atrial mechanical failure.

M MODE ECHOCARDIOGRAM

Mean values of left ventricular cavity size were increased and fractional shortening reduced in patients with left atrial mechanical failure (table 1). Isovolumic relaxation time was short, being $<40 \mathrm{~ms}$, the lower $95 \%$ limit of normal, in all patients. The left atrial dimension was consistently increased except in the single patient who had undergone atrial resection, in whom it was $2.0 \mathrm{~cm}$. The ventricular systolic amplitude of right and left AV rings was lower than normal. Motion of the tricuspid ring with right atrial systole was detected in nine patients including the one with a DDD pacemaker, though the mean value of systolic amplitude was reduced. Atrial mechanical activity at the septum was absent in all but one patient in whom a deflection of $1 \mathrm{~mm}$ was identified.

\section{DOPPLER ECHOCARDIOGRAM}

The mean value of transmitral $E$ wave peak velocity was slightly increased in patients with left atrial mechanical failure, and deceleration time was consistently short, being $<120 \mathrm{~ms}$ in seven patients (table 1). ${ }^{2}$ Mild functional mitral regurgitation was present in eight patients. Transtricuspid flow velocity patterns were normal.

ELECTROCARDIOGRAM

Nine patients were in sinus rhythm, by definition, with normal $P$ wave voltage and vector (table 2). The $P$ wave was widened in two

Table 2 Electrocardiographic data in 10 patients

\begin{tabular}{lc}
\hline Indices & Mean $(S D)$ \\
\hline P wave axis $\left({ }^{\circ}\right)$ & $63(19)$ \\
P wave voltage $(\mathrm{mV})$ & $0 \cdot 1(0 \cdot 03)$ \\
P wave width (ms) & $103(21)$ \\
PR interval (ms) & $177(37)$ \\
RR interval (ms) & $825(160)$ \\
\hline
\end{tabular}


patients. PR interval was prolonged in three patients with values ranging from 208 to 243 $\mathrm{ms}$. The AV interval was programmed to 200 $m s$ in the patient with a DDD pacemaker.

\section{Discussion}

These observations suggest that the left atrium may be effectively paralysed in some patients despite the presence of normal sinus rhythm. Most patients had a dilated ventricle with a short $\mathrm{E}$ wave deceleration time suggesting restrictive filling. ${ }^{23}$ There were no characteristic features of the standard 12 lead ECG.

Left atrial paralysis must be distinguished from severe restrictive left ventricular disease, where greatly increased end diastolic cavity stiffness effectively prevents ventricular volume increasing during atrial systole. A waves are thus absent on left ventricular long axes and transmitral Doppler, but are characteristically increased on the apexcardiogram (fig 3(A)). ${ }^{24}$ The apexcardiogram closely reflects ventricular pressure during diastole. ${ }^{5}$ The combination of an increased pressure $A$ wave with a reduced volume change defines low end diastolic ventricular compliance. By contrast, pressure and volume changes were absent in the present study (figs 2 and 3(B)).

The main limitation of this study is the extent to which severely reduced atrial function can be distinguished from its complete absence. Measurements made by transmitral Doppler used a wall filter of $5 \mathrm{~cm} . \mathrm{s}^{-1}$; atrial flow velocities less than this value may have been present. Elongation of the left ventricular long axis during atrial systole was $<1 \mathrm{~mm}$, a value determined by the depth resolution of the echocardiograph. The diagnosis of atrial paralysis depends critically on recording a technically satisfactory apexcardiogram, as otherwise the condition cannot be distinguished from restrictive ventricular disease. This is not always possible, so other cases may have been missed. Restrictive ventricular disease may be accompanied by retrograde flow in the pulmonary veins coinciding with atrial systole. Measurement of such flow by transthoracic echocardiography is at the limits of technical feasibility, particularly if left atrial or left ventricular cavity size is increased, so failure to record this feature would lack conviction as a major diagnostic criterion. In the absence of clinical indication we did not feel justified in submitting our patients to transoesophageal echocardiography.

Left atrial paralysis might theoretically result from failure of electrical activation. Although we did not record the left atrial electrogram directly, we noted that all our patients were in sinus rhythm and that normal right atrial contraction was present in all but one. None of our patients showed the ECG features of complete interatrial block. ${ }^{6}$ There was no evidence of isolated left atrial fibrillation $^{78}$ nor atrial standstill, which has been sporadically recorded. ${ }^{9-10}$ We did not subject our patients to coronary arteriography, so we cannot definitively exclude atrial infarction. This seems unlikely in view of the uniformity and completeness of the loss of atrial function and the variability of atrial blood supply.

The closest resemblance to our findings seems to be those of Plehn et al ${ }^{11}$ who described a single patient with cardiac amyloid in whom pressure and transmitral Doppler evidence of left atrial activity was absent despite the presence of sinus rhythm and a right atrial A wave. None of our patients had amyloid disease. Although most patients had a dilated left ventricle with restrictive filling, they were not as severe as that described in a group of patients in whom the apexcardiographic A waves were conspicuously increased. ${ }^{2}$ Intact atrial function would thus have been expected. Left atrial cavity size was indeed increased, but the usual response to left atrial enlargement is fibrillation rather than mechanical standstill. Loss of an A wave on the apexcardiogram occurs with severe mitral stenosis, which was not present in any of our patients, but atrial pressure transients are particularly well conducted to the apex in restrictive ventricular disease.

Loss of left atrial mechanical activity has practical consequences. Transmitral Doppler flow velocity pattern, as reflected in the $\mathrm{A} / \mathrm{E}$ ratio, is widely used to assess left ventricular diastolic function. In particular a reduction in peak $A$ wave velocity is taken as evidence of restrictive cardiomyopathy. This conclusion would have been inappropriate in the patients we have described. In addition, the fact that there may be complete loss of atrial systolic function raises the possibility that partial loss of atrial function may occur in other patients. This loss might be expected to reduce transmitral A wave velocities independently of any ventricular diastolic disease. A method that fails to distinguish between atrial systolic and ventricular diastolic function is likely to be unsatisfactory. Functional consequences to the patients seem to have been minor. All patients except the one with the DDD pacemaker had the advantage of being in sinus rhythm, so a physiological rate response to exercise and a regular rhythm would have been expected. There is no increment in ventricular stroke volume with atrial systole in patients with more severe restrictive disease, though the raised A wave merely increased mean left atrial pressure and caused retrograde flow of blood back up the pulmonary veins.

Left atrial mechanical failure may thus accompany left ventricular disease in a few cases. Little is known of the untreated course of the condition: whether it progresses to fibrillation, whether it predisposes to stagnation (and should thus be regarded as an embolic risk), or whether it has any prognostic significance. As criteria for its diagnosis have been defined, however, this information can now be collected.

We thank Mr Ugo Pastorino for permission to report a case operated on by him. K W is supported by The Royal Brompton Hospital Special Cardiac Fund. 
1 Jones CJH, Song GJ, Gibson DG. An echocardiographic assessment of atrial mechanical behaviour. Br Heart $\mathcal{F}$ 1991;65:31-6.

2 Henein MY, Gibson DG. Abnormal subendocardial function in restrictive left ventricular disease. Br Heart $f$ 1994;72:237-42.

3 Appleton CP, Hatle LK, Popp RL. Demonstration of restrictive ventricular physiology by Doppler echocardiorestrictive ventricular physiology by Dopple

$4 \mathrm{Ng} \mathrm{KSK}$, Gibson DG. Relation of filling pattern to diastolic function in severe left ventricular disease. Br Heart $\mathcal{F}$ 1990;63:209-14.

5 Denef B, Van de Werf F, Geest HD, Kesteloot H. Calibrated apexcardiography and assessment of left ventricular dynamics in man. Eur $\mathcal{F}$ Cardiol 1976;4(suppl): 143-52.

6 Bayes de Luna A, Fort de Ribot R, Trilla E, Julia J, Garcia
J, Sadurni J, et al. Electrocardiographic and vectorcardiographic study of interatrial conduction disturbances with left atrial retrograde activation. F Electrocardiol 1985;18:1-14.

7 Chung EK. Atrial dissociation due to unilateral atrial fibrillation. $\mathcal{F}$ Electrocardiol 1969;2:373-6.

8 Chung EK. Manual of cardiac arrhythmias. Stoneham, Massachusetts: Yorke Medical Books, 1986:289-92.

9 Amram SS, Vagueiro MC, Pimenta A, Machado HB. Persistent atrial standstill with atrial inexcitability. $P A C E$ 1978;1:80-9.

10 Maeda S, Tanaka T, Hayashi T. Familial atrial standstill caused by amyloidosis. $\mathrm{Br}$ Heart $\mathcal{f} 1988 ; 59$ : 498-500.

11 Plehn JF, Southworth J, Cornwell III GG. Brief report: atrial systolic failure in primary amyloidosis. $N$ Engl $f$ Med 1992;327:1570-3.

\section{IMAGES IN CARDIOLOGY}

\section{Infective endocarditis}

These are the hands and feet in a patient who presented with a pyrexia and in whom aortic incompetence was noted. The echocardiogram confirmed the diagnosis of endocarditis with a large vegetation on the aortic valve. Blood cultures grew Staphylococcus aureus. Multiple Janeway lesions were present on the thenar and hypothenar eminences of the hand, the sole of the foot, the tips of the fingers, and the plantar surfaces of the toes. As seen in cases of acute valve infection some of these lesions were haemorrhagic

M K DAVIES 